\title{
Overview of the MicroBooNE LArTPC Detector Calibration
}

Wanwei Wu, Fermilab (on behalf of the MicroBooNE Collaboration) APS April Meeting 2019, Denver, Colorado 


\section{The MicroBooNE Experiment}

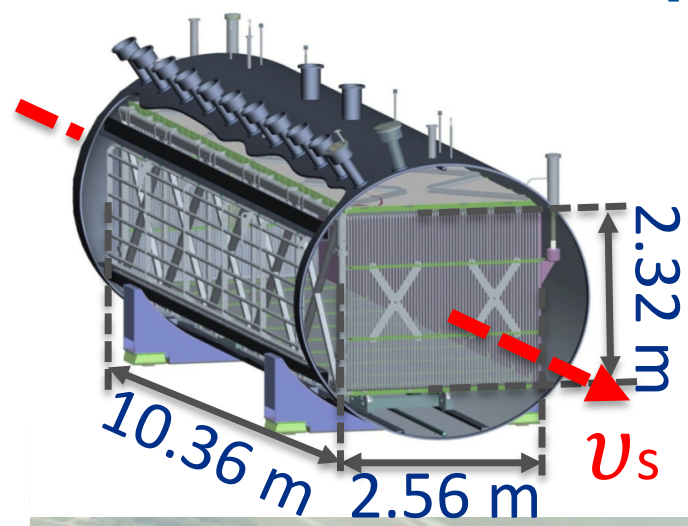

- Liquid Argon Time Projection Chamber (LArTPC)

- Investigate low-energy excess (LEE) of electron-like neutrino events observed by MiniBooNE in 2009

- Measure the low energy neutrino cross sections

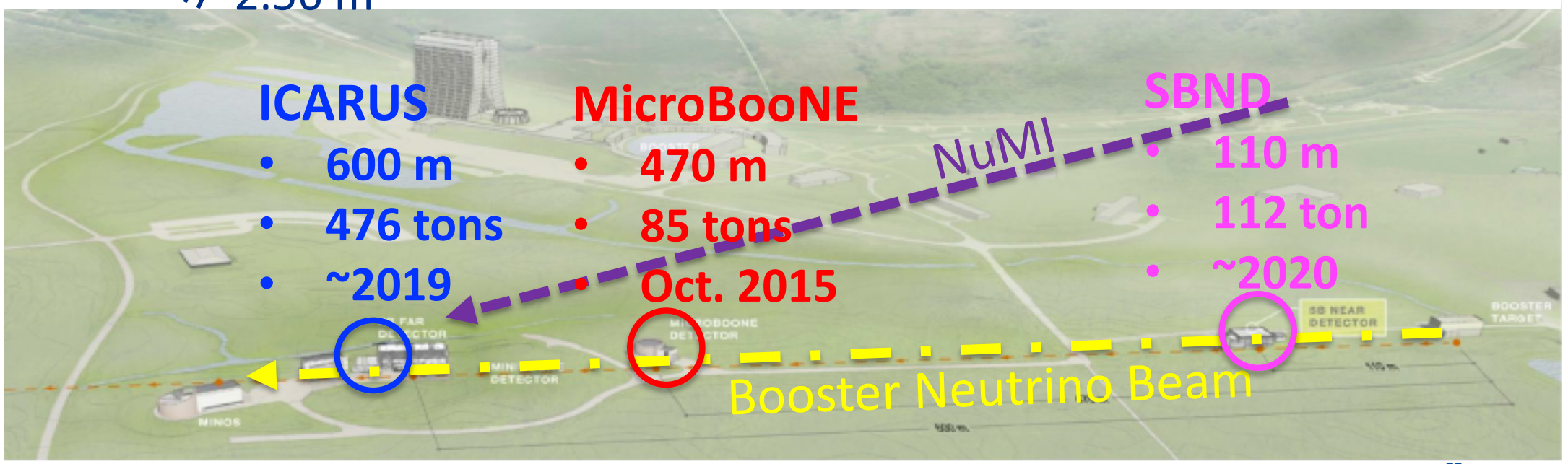




\section{The MicroBooNE Experiment}
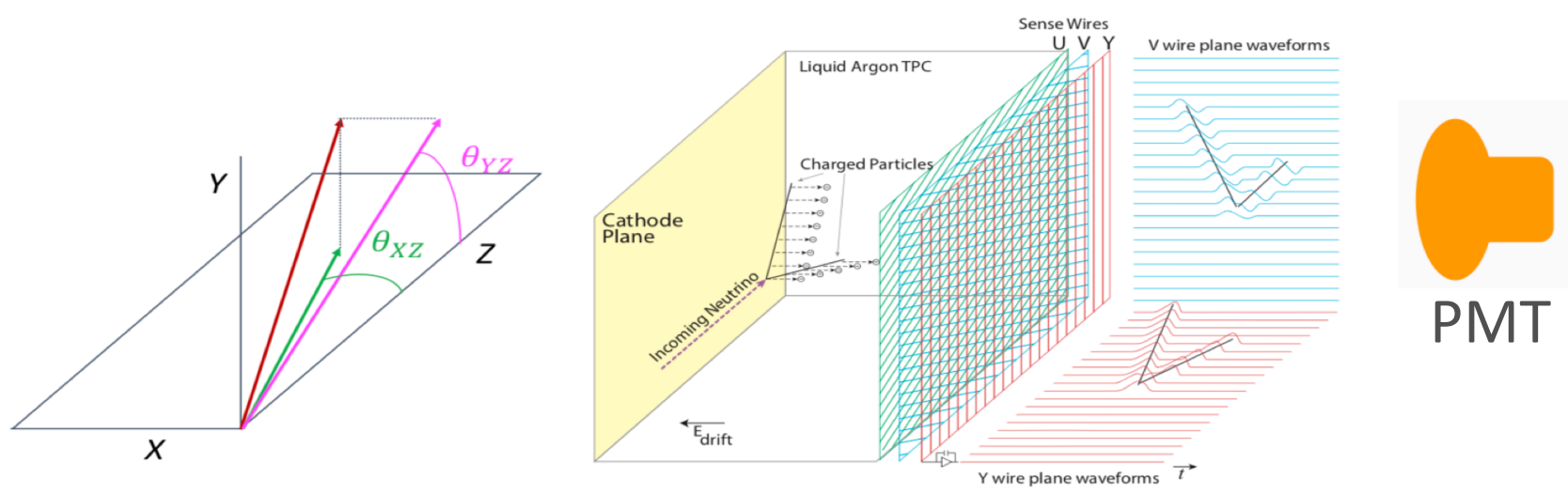

- 3 wire planes:

- separated by $3 \mathrm{~mm}$, with $3 \mathrm{~mm}$ wire spacing for each

- to reconstruct the event, tracking and calorimetry

- 32 PMTs:

- to detect scintillation light, mainly for trigger and event selection

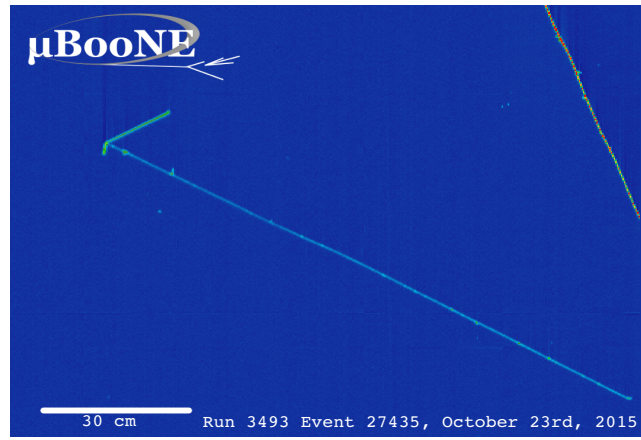




\section{Example of Simulated Digitized Signals}

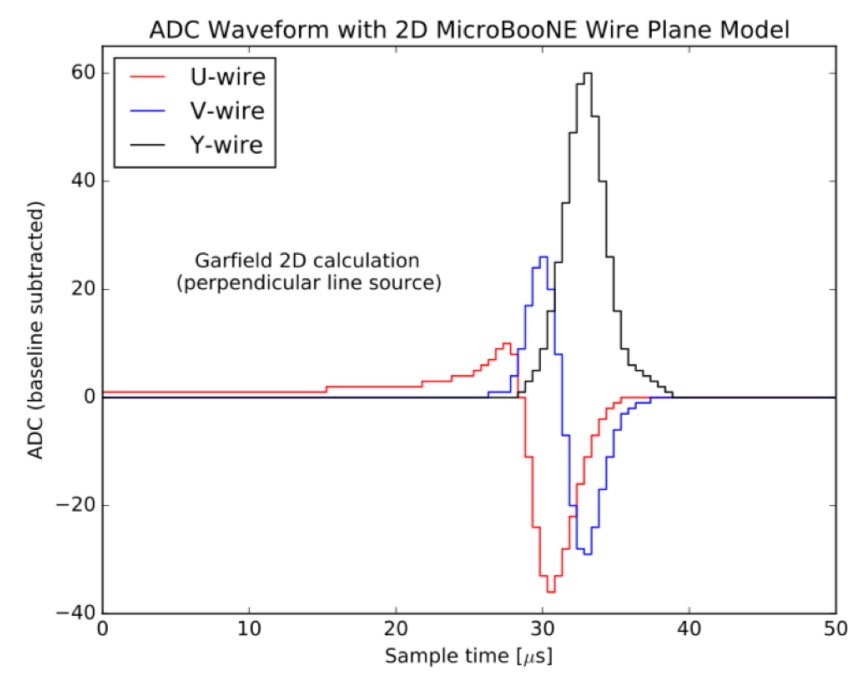

(ADC: Analog-to-digital converter)

The digitized signals from a central wire from each plane that are induced by an ideal MIP track in a 2D model of the MicroBooNE TPC.

----Refer: JINST 12, P08003 (2017) 


\section{Reconstruction of Drifted Electrons}
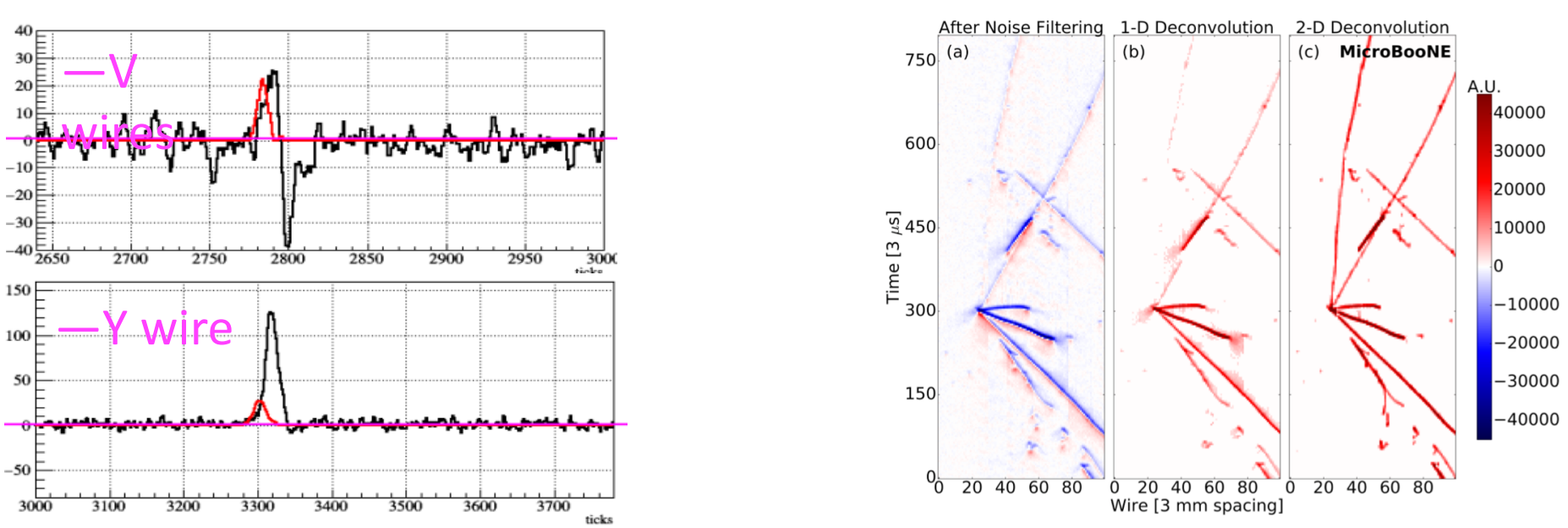

----Image from W. Gu

----Refer: JINST 13, P07006 (2018)

- We use deconvolution to extract original signals:

- Noise filtering \& Signal processing

- Deconvolution is a mathematical technique to extract the original signal $S(t)$ from the measured signal $M\left(t^{\prime}\right)$.

$$
M\left(t^{\prime}\right)=\int_{-\infty}^{\infty} R\left(t, t^{\prime}\right) \cdot S(t) d t
$$$$
R\left(t, t^{\prime}\right) \text { : detector response function }
$$ 


\section{Detector Calibration}

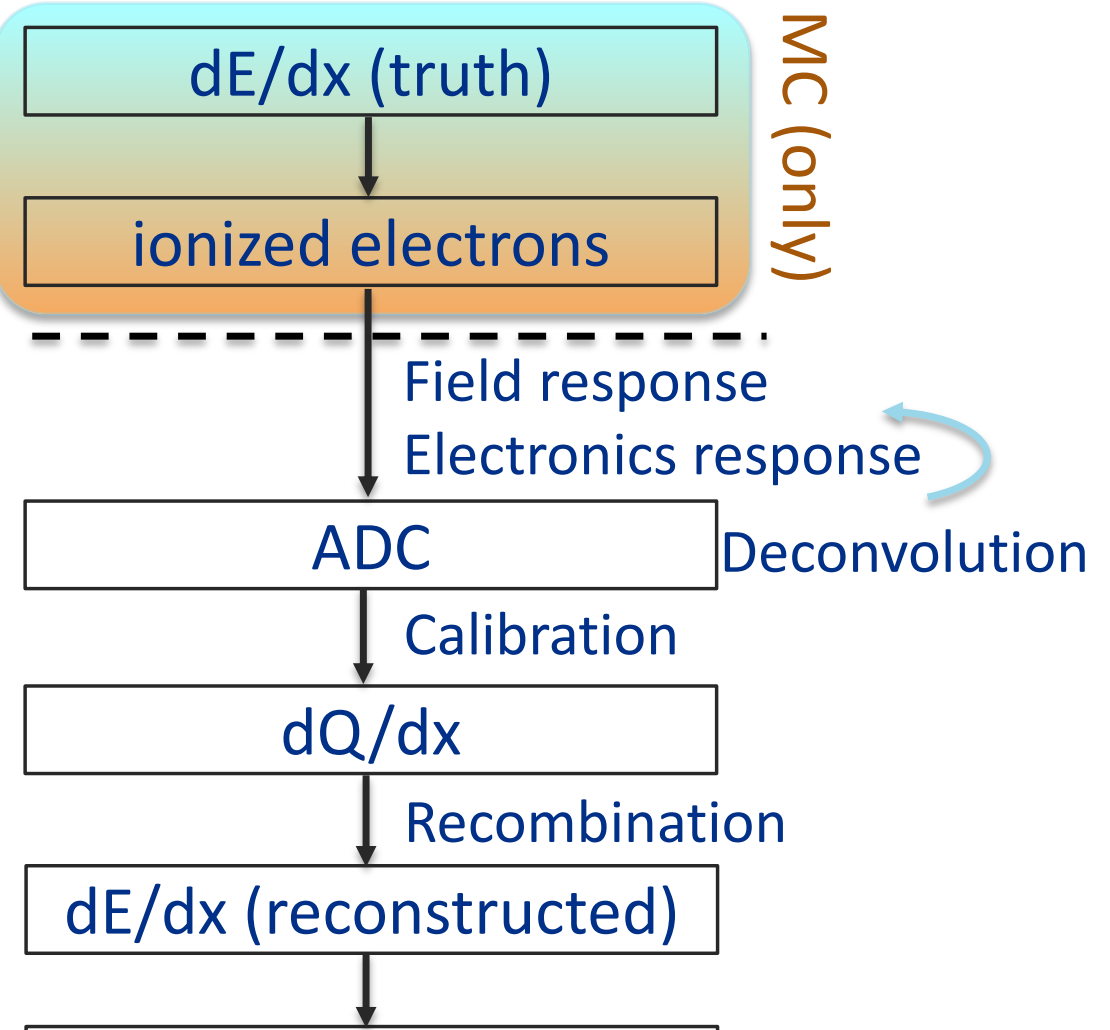

Many other effects need to be calibrated:

I. Detector uniformity calibration $(\mathrm{dQ} / \mathrm{dx})$

- Variation in electronics gains

- Electron attenuation (e lifetime)

- Nonuniform E field due to Space Charge Effects (SCE), diffusion, disconnected wires, TPC edges, etc.

- Temporal variations: i.e., change of run condition, temperature

II. Energy scale determination ( $\mathrm{dE} / \mathrm{dx}$ )

Particle Identification

- Use the well-known energy loss profile 


\section{Space Charge Effects}

- MicroBooNE detector is on the surface ( 20-30 cosmic muons per 4.8 ms readout window)

- Distortion of E field and ionization drift trajectories due to accumulation of slow-moving argon ions produced from cosmic muons impinging TPC

- Calibration on the E-field can be done by using UV laser system and the cosmic muon tracks

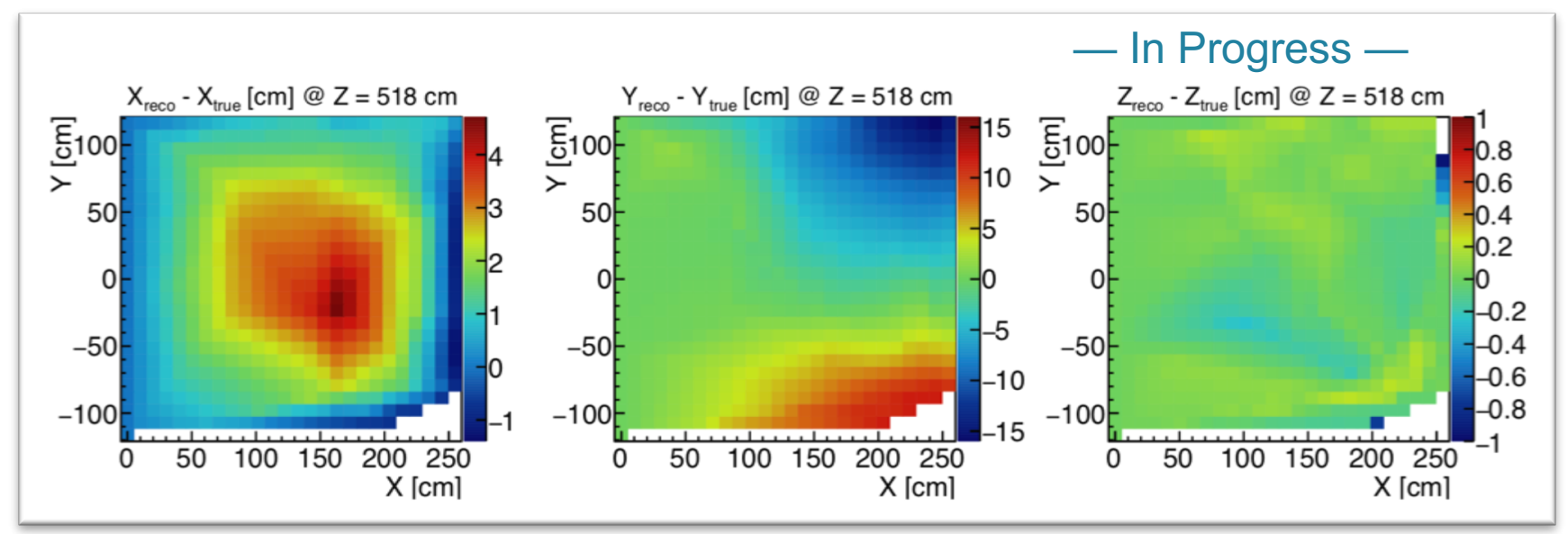

At central $Z$ the spatial distortion of $d X=X_{\text {reco }}-X_{\text {true }}$ (left), $d Y=Y_{\text {reco }}-Y_{\text {true }}$ and $d Z=Z_{\text {reco }}-Z_{\text {true }}$ from laser data. 


\section{Detector Uniformity Calibration- $d \mathrm{Q} / \mathrm{dx}$}

- $d Q / d x$ calibration ( $d Q / d x$ : ionization charge per unit length)

- make the detector response to ionization charge uniformly throughout the detector and in time: $Y Z$ plane, $X$ direction, $T$

- use anode-cathode crossing cosmic muons to cover whole drift distance

- Variation of detector response $(\mathrm{dQ} / \mathrm{dx}$ ) in $\mathrm{YZ}$ plane and in the drift direction $\mathrm{X}$

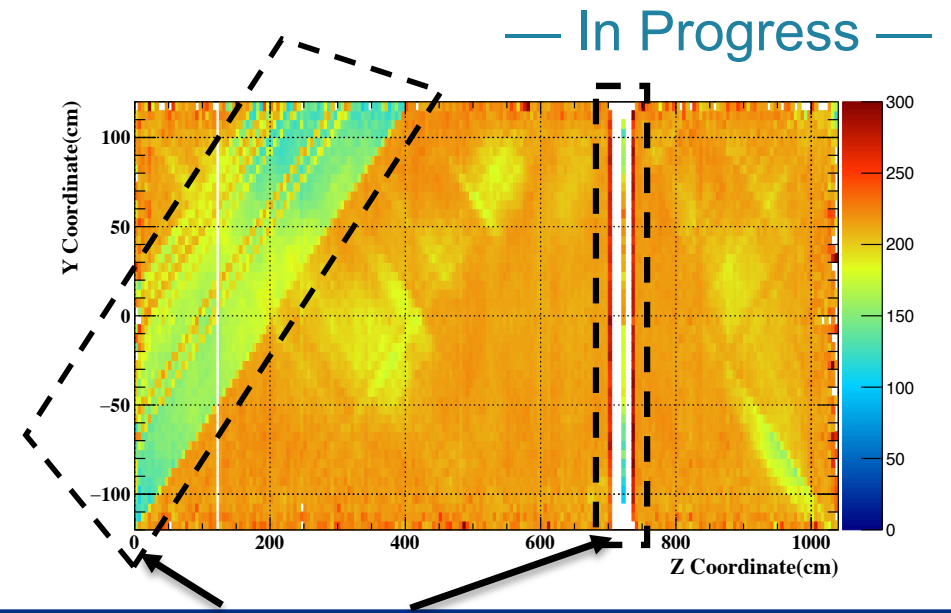

Misconfigured/cross-connected TPC channels
- In Progress -

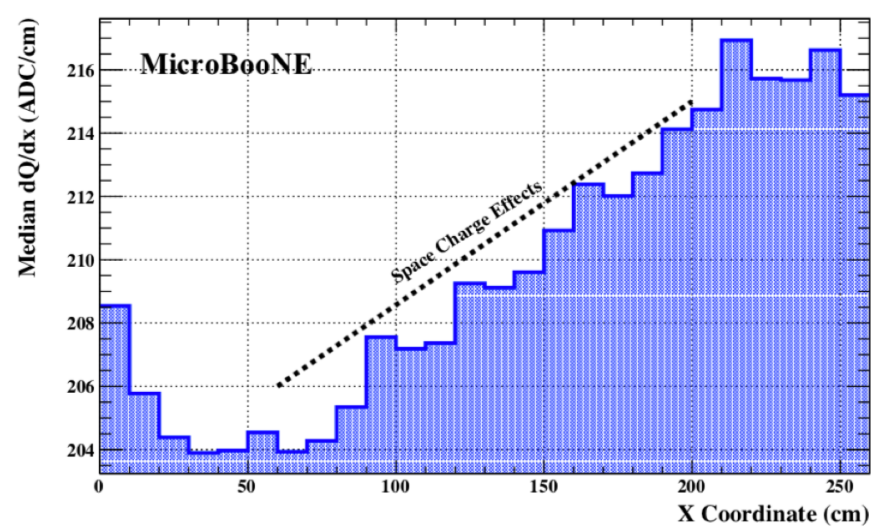

----Refer: MICROBOONE-NOTE-1048-PUB 


\section{Detector Uniformity Calibration- $\mathrm{dQ} / \mathrm{dx}$}

- Results (MC does not have time dependence):

- Data:

$$
(d Q / d x)_{\text {corr }}^{\text {data }}=C(t) \cdot C(x) \cdot C(y, z) \cdot(d Q / d x)_{\text {uncorr }}
$$

- Monte Carlo (MC): $(d Q / d x)_{\text {corr }}^{M C}=C(x) \cdot C(y, z) \cdot(d Q / d x)_{\text {uncorr }}$

- Comparisons between calibrated and uncalibrated $\mathrm{dQ} / \mathrm{dx}$ for both $\mathrm{MC}$ and data
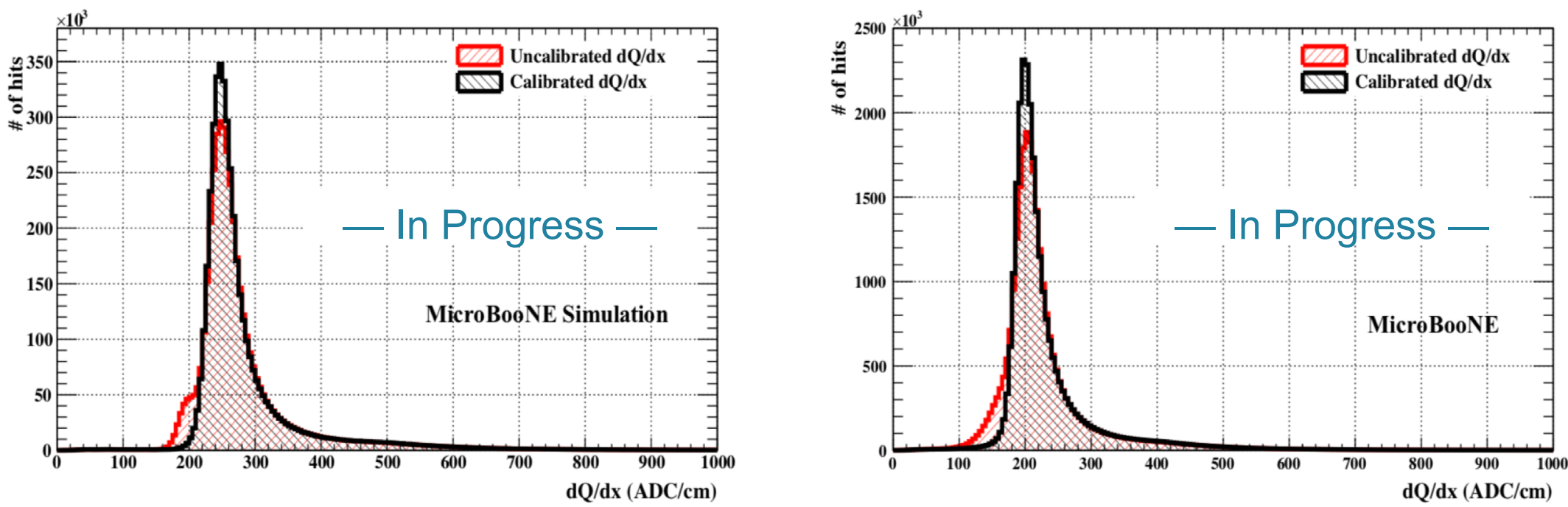


\section{Energy Scale Determination-dE/dx}

- Determine the calibration constant $C$ (unit: $A D C / e)$, which translates the corrected $\mathrm{dQ} / \mathrm{dx}(\mathrm{ADC} / \mathrm{cm})$ to $\mathrm{dQ} / \mathrm{dx}(\mathrm{e} / \mathrm{cm})$.

- Stopping muons from neutrino interactions or cosmic rays are used to study the measured and predicted Most Probable $\mathrm{dE} / \mathrm{dx}$ Value (MPV):

$$
\left(\frac{d E}{d x}\right)_{c o r r .}=\frac{\exp \left(\frac{\left(\frac{d Q}{d x}\right)_{c o r r} .}{C} \cdot \frac{\beta^{\prime} W_{i o n}}{\rho \xi}\right)-\alpha}{\frac{\beta^{\prime}}{\rho \xi}}
$$

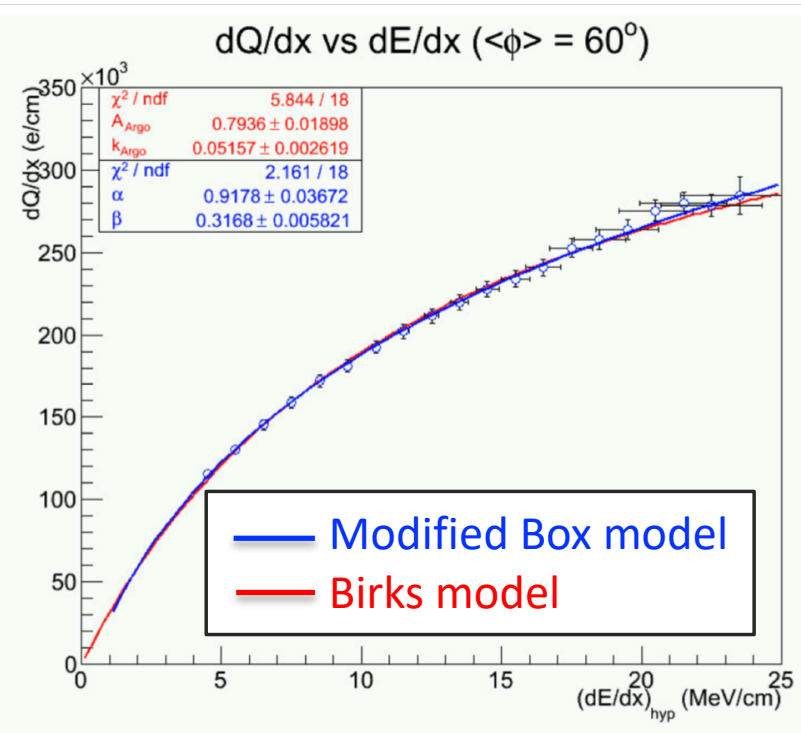

----Refer: ArgoNeuT JINST 8, P08005 (2013) 


\section{Energy Scale Determination-dE/dx}

- Once we find the calibration constants, we would expect the calibrated $\mathrm{dE} / \mathrm{dx}$ matches expectation.

- In Progress -

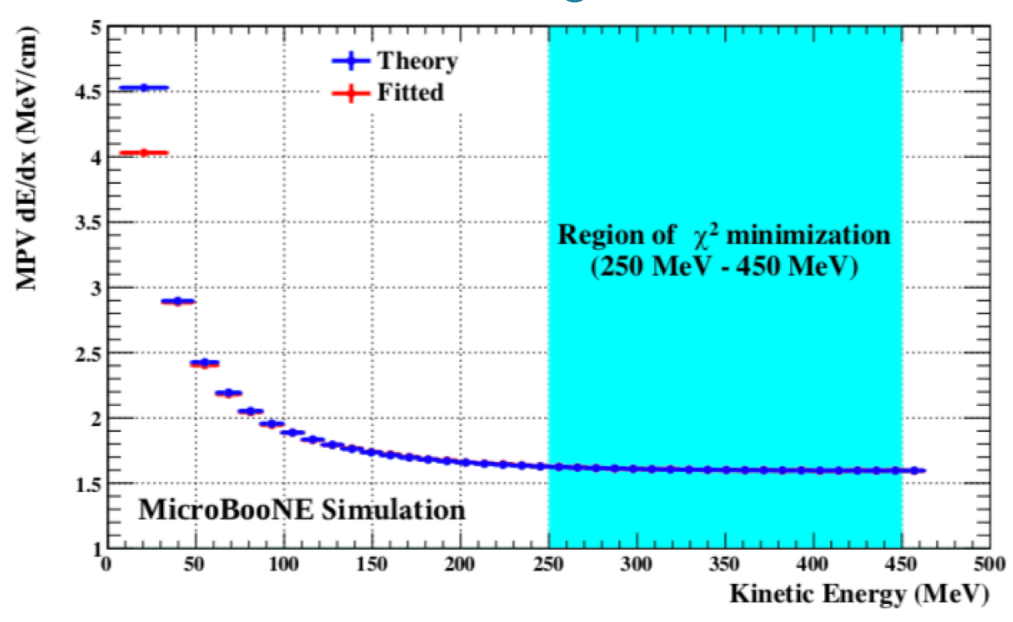

- In Progress —

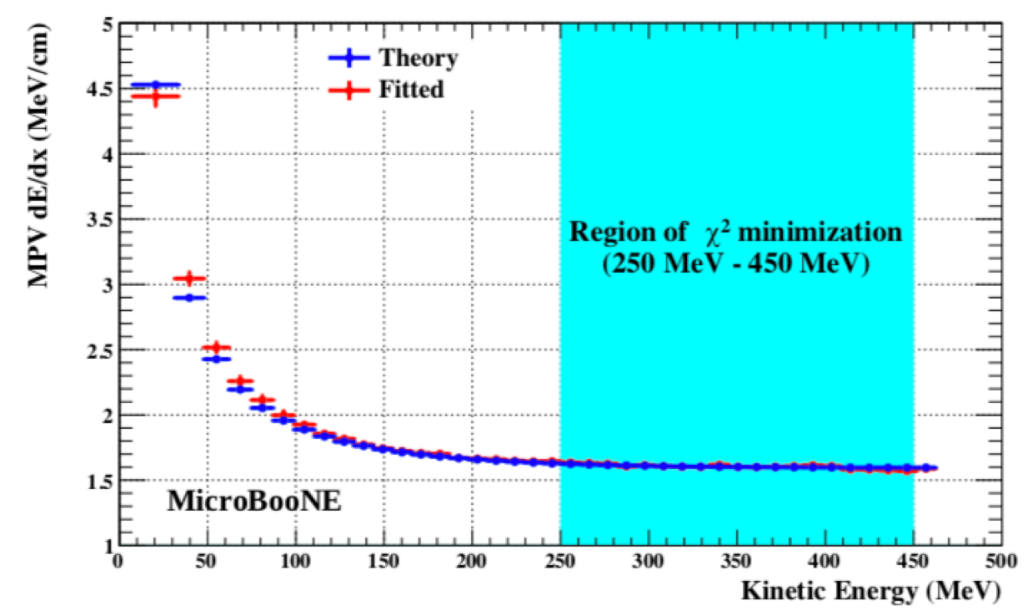

- Kinetic Energy (K.E.) $=\sum \frac{d E}{d x} d x$, where $d x$ is the track pitch. 


\section{Summary}

- In this talk, we present a briefly overview of the MicroBooNE LArTPC detector calibration.

- MicroBooNE detector performs at a high signal-to-noise ratio.

- Deconvolution is used to extract the charge signal after removing noise.

- Many detector effects will affect the measurement and need to be addressed appropriately.

- The detector calibration can correct most of the detector effects and improve the energy reconstruction and particle identification.

- The calibration strategy carried out here can be used by other LArTPC experiments to calibration their detectors. 


\section{Acknowledgement}

- The calibration method is developed by the MicroBooNE Calibration Group. I would like to express my gratitude and appreciation to the team, especially Tingjun Yang, Glenn Horton-Smith, Mike Mooney, Yifan Chen, Varuna Meddage and Marianette Wospakrik. Without their effort and help, this talk would not have been possible.

- MicroBooNE is supported by the following: the U.S. Department of Energy, Office of Science, Offices of High Energy Physics and Nuclear Physics; the U.S. National Science Foundation; the Swiss National Science Foundation; the Science and Technology Facilities Council of the United Kingdom; and The Royal Society (United Kingdom).

-Thank you! 


\section{Backup-Fermilab Neutrino Programs}

\section{Fermilab Neutrino Experiments}

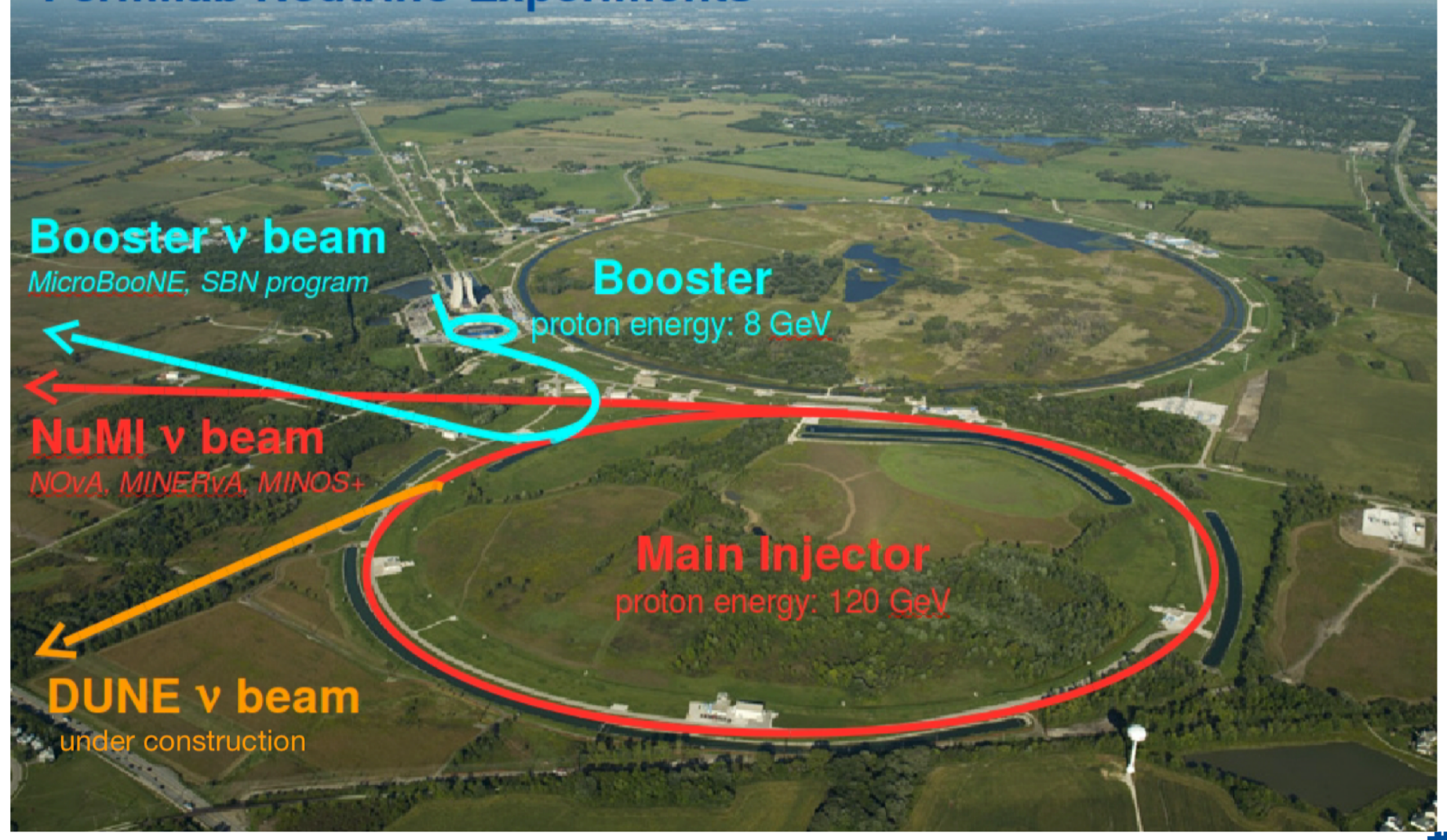




\section{Backup-PMT and Trigger Systems}

- 32 TPB-coated PMTs behind wires

- Two gains: $1.8 \%$ and $18 \%$

- Shaping: 60 ns rise time

- $64 \mathrm{MHz}$ ADC (ADS5272): accurate determination of event $t_{0}$

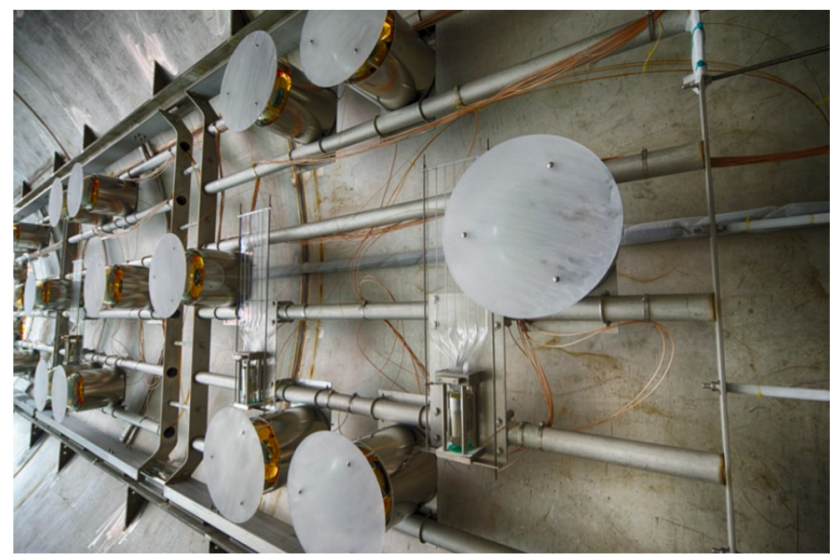

- Level-1 trigger: accelerator gates (BNB and NuMI) and random triggers (for cosmics)

- Level-2 trigger: PMT information in the beam window (in software)

MicroBooNE: JINST 10, T06001 (2015); JINST 8, T07005 (2013) 


\section{Backup-Drift Electron Path}
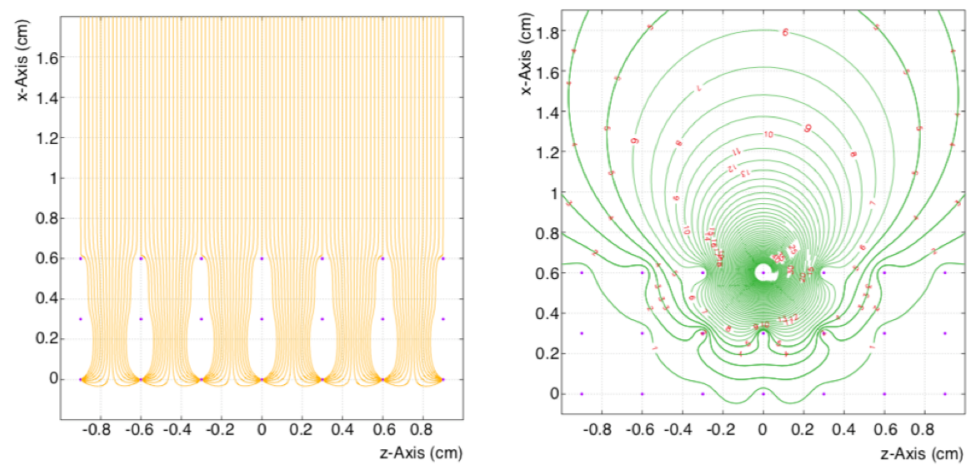

(a) Electron drift paths.

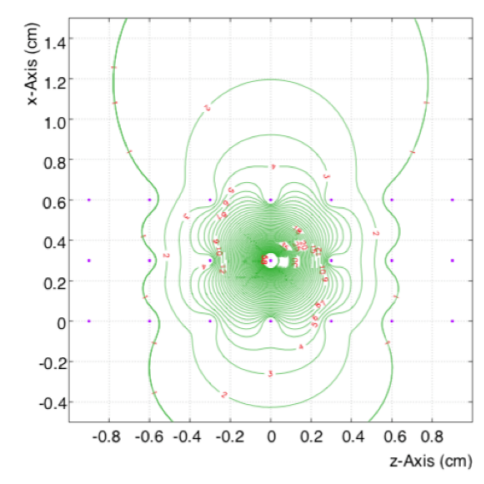

(b) Weighting potential on a $\mathrm{U}$ wire.

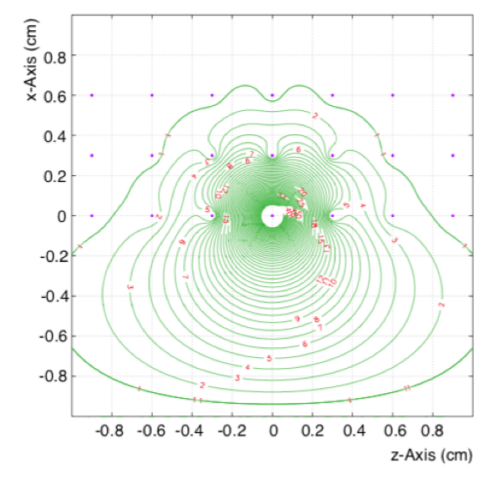

----Refer: JINST 13, P07006 (2018)

(c) Weighting potential on a $\mathrm{V}$ wire.

(d) Weighting potential on a $\mathrm{Y}$ wire. 


\section{Backup-Deconvolution}

The principal method to extract charge is deconvolution. This procedure in its one-dimensional (1D) form has been used in the data analysis of previous liquid argon experiments [29, 30]. This technique has the advantages of being robust and fast. It is an essential step in the overall driftedcharge profiling process.

Deconvolution is a mathematical technique to extract the original signal $S(t)$ from the measured signal $M\left(t^{\prime}\right)$. The measured signal is modeled as a convolution integral over the original signal $S(t)$ and a given detector response function $R\left(t, t^{\prime}\right)$, which gives the instantaneous portion of the measured signal at some time $t^{\prime}$ due to an element of original signal at time $t$ :

$$
M\left(t^{\prime}\right)=\int_{-\infty}^{\infty} R\left(t, t^{\prime}\right) \cdot S(t) d t
$$




\section{Backup-Noise Filter}

Raw Waveform

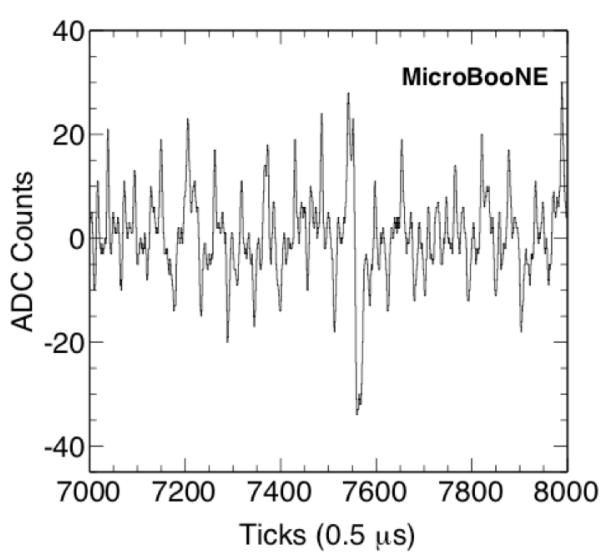

----Refer: JINST 12, P08003 (2017)
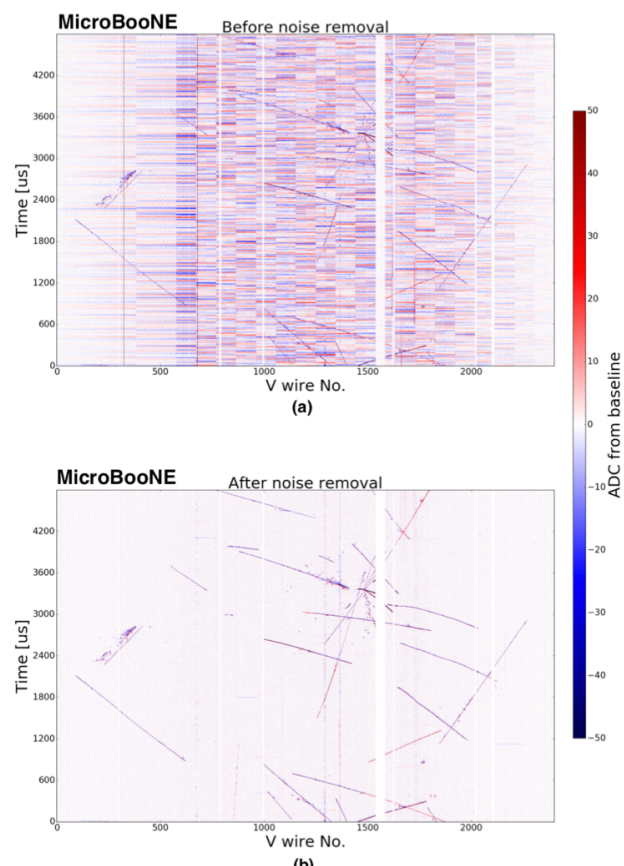

(b)

Figure 14. 2-D event display of the $\mathrm{V}$ plane from run 3493 event 41075 showing the raw signal (a) before and (b) after offline noise filtering. A clean event signature is recovered once all the identified noise sources are subtracted. 


\section{Backup-MicroBooNE Calibration}

- Challenges for MicroBooNE LArTPC detector:

- Misconfigured or cross-connected TPC channels: change gain of electronic channels, distort field between wire planes

- Space Charge Effects (SCE): cosmic rays, accumulation of slow moving $\mathrm{Ar}^{+}$, causing distortion the magnitude and direction of drift $E$ field

- Electron attenuation: electronegative contaminants such as $\mathrm{H} 2 \mathrm{O}$ and $\mathrm{O} 2$ can capture some of the drifting electrons

- Diffusion: drifted electrons may get smeared out

- Recombination: ionization electrons may not completely liberate from their parent argon ions and recombine back to form neutral argon atoms again, causing underestimation of particle energy loss

- Temporal variations: change of temperature, run conditions...

- We want to measure the charge and position of ionization signal precisely and improve both the measurement of total deposited energy and particle identification (PID). 


\section{Backup-Space Charge Effects}

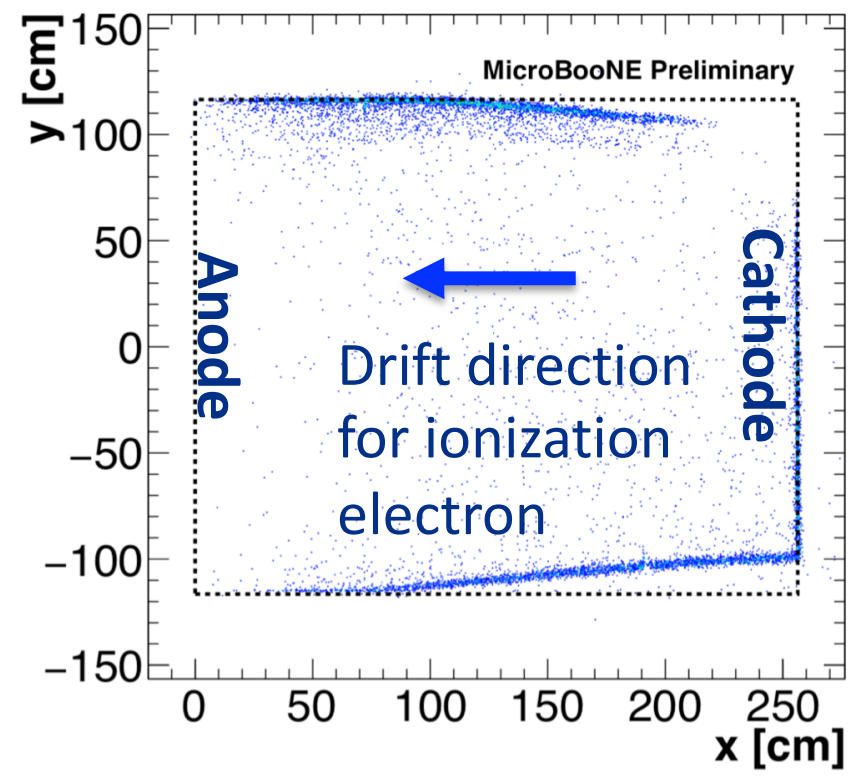

Start/end points of reconstructed cosmic muon tracks tagged by an external muon counter in the $x$-y plane for off-beam (cosmic) events.

----Refer: MICROBOONE-NOTE-1018-PUB 


\section{Backup-dQ/dx}

$>\left(\frac{d Q}{d x}\right)_{\text {uncorr. }} \rightarrow Y Z_{\text {corr. }} \rightarrow$ DriftDirection $_{\text {corr. }} \rightarrow$ Time $_{\text {corr. }} \rightarrow\left(\frac{d Q}{d x}\right)_{\text {corr. }}$

- Consists of 3 steps:

1. YZ plane correction: remove effects from SCE, misconfigured or cross-connected TPC channels and transverse diffusion

2. Drift direction correction: remove effects coming from electron attenuation, SCE and longitudinal diffusion

3. Time correction: remove any temporal variations in detector response (for data only, MC does not have time dependence)

$\left(\frac{d Q}{d x}\right)_{\text {uncorr. }} \rightarrow Y Z_{\text {corr. }} \rightarrow$ Lifetime $_{\text {corr }} \rightarrow\left(\frac{d Q}{d x}\right)_{\text {corr }}$

- Consists of 2 steps:

1. YZ plane correction: same as above

In progress

2. Lifetime correction:

- remove effects coming from electron attenuation, SCE and longitudinal diffusion, temporal variations

- $Q=Q^{\prime} \cdot e^{-\left(\frac{t}{\tau}\right)}$ (t: drift time; $\tau$ : electron lifetime, determined by purity or TPC (laser system, muon count system ) 


\section{Backup-dQ/dx: YZ Plane Correction}

The YZ plane is segmented into $5 \mathrm{~cm}$ by $5 \mathrm{~cm}$ cells. The correction factor is defined by:

$$
C(y, z)=\frac{(d Q / d x)_{\text {Global }}}{(d Q / d x)_{\text {Local }}}
$$

$(d Q / d x)_{G l o b a l}$ - Global median $d Q / d x$ in the collection plane

$(d Q / d x)_{\text {Local }}$ - Local median $d Q / d x$ for a given YZ plane cell in the collection plane 


\section{Backup-dQ/dx: Drift Direction}

After YZ plane calibration:

$$
(d Q / d x)_{\text {corr. }}=C(y, z) \cdot(d Q / d x)_{\text {uncorr }} .
$$

The drift direction is segmented into $10 \mathrm{~cm}$ in data $(5 \mathrm{~cm}$ in $\mathrm{MC})$. The correction factor is defined by:

$$
C(x)=\frac{(d Q / d x)_{\text {Global }}^{\prime}}{(d Q / d x)_{\text {Local }}^{\prime}}
$$

$(d Q / d x)_{\text {Global }}^{\prime}$ - Global median $d Q / d x$ value after correcting for YZ plane irregularities

$(d Q / d x)_{\text {Local }}^{\prime}$ - Local median $d Q / d x$ value after correcting for YZ plane irregularities 


\section{Backup-dQ/dx: Time Calibration}

After YZ plane calibration and drift direction calibration:

$$
(d Q / d x)_{\text {corr }}=C(x) \cdot C(y, z) \cdot(d Q / d x)_{\text {uncorr }} .
$$

The time dependent correction is determined for each day:

$$
C(t)=\frac{(d Q / d x)_{\text {Reference }}}{(d Q / d x)_{\text {Global }}}
$$

$(d Q / d x)_{\text {Reference }}$ - Selected reference $d Q / d x$ value $(d Q / d x)_{\text {Global }} \quad-$ Global $d Q / d x$ value in the anode wire plane after correcting for YZ plane and drift direction irregularities 


\section{Backup-dE/dx}

$$
\left(\frac{d E}{d x}\right)_{c o r r .}=\frac{\exp \left(\frac{\left(\frac{d Q}{d x}\right)_{c o r r}}{C} \cdot \frac{\beta^{\prime} W_{\text {ion }}}{\rho \xi}\right)-\alpha}{\frac{\beta^{\prime}}{\rho \xi}}
$$

- $\quad C$ : Calibration constant to convert ADC values to number of electrons

- $W_{\text {ion }}: 23.6 \times 10^{-6} \mathrm{MeV} /$ electron (work function of argon)

- $\quad \xi: 0.273 \mathrm{kV} / \mathrm{cm}$ (MicroBooNE drift electric field)

- $\rho: 0.212(\mathrm{kV} / \mathrm{cm})\left(\mathrm{g} / \mathrm{cm}^{2}\right) / \mathrm{MeV}$

- $\quad \alpha: 0.93$

\} ArgoNeuT: JINST 8, P08005 (2013)

$$
\chi^{2}=\Sigma\left(\frac{\left(M P V(d E / d x)_{\text {prediction }}-M P V(d E / d x)_{\text {Measured }}\right)^{2}}{\sigma^{2}}\right)
$$

